September 23, 2013

LBNL-41152, UCB-PTH-97/61, NSF-ITP-98-002

hep-th/9802005

\title{
Membrane Scattering in Curved Space with M-Momentum Transfer
}

\author{
Jan de Boer ${ }^{1,2}$, Kentaro Hori ${ }^{1,2,3}$, and Hirosi Ooguri ${ }^{1,2,3}$ \\ ${ }^{1}$ Department of Physics, University of California \\ Berkeley, CA 94720-7300, U.S.A. \\ ${ }^{2}$ Ernest Orlando Lawrence Berkeley National Laboratory \\ Mail Stop 50A-5101, Berkeley, CA 94720, U.S.A. \\ ${ }^{3}$ Institute for Theoretical Physics, University of California \\ Santa Barbara, CA 93106, U.S.A
}

\begin{abstract}
We study membrane scattering in a curved space with non-zero M-momentum $p_{11}$ transfer. In the low-energy short-distance region, the membrane dynamics is described by a three-dimensional $N=4$ supersymmetric gauge theory. We study an $n$-instanton process of the gauge theory, corresponding to the exchange of $n$ units of $p_{11}$, and compare the result with the scattering amplitude computed in the low-energy long-distance region using supergravity. We find that they behave differently. We show that this result is not in contradiction with the large- $N$ Matrix Theory conjecture, by pointing out that cutoff scales of the supergravity and the gauge theory are complementary to each other.
\end{abstract}




\section{Introduction}

There are three basic length scales in M Theory compactified on a circle [1]: the IIA string length $l_{s}$, the Planck length $l_{p}=g^{1 / 3} l_{s}$ and the radius of the M-circle $R_{s}=g l_{s}$, with $g$ being the IIA string coupling constant. In the weak coupling region $g \ll 1$ of the IIA string, the three scales line up as

$$
R_{s} \ll l_{p} \ll l_{s}
$$

In the low-energy short-distance region, the dynamics of $\mathrm{D}$ (irichlet) branes [2] is described by a gauge theory on the D-brane worldvolume [3-6]. In flat space, the $v^{4}$-term in the scattering amplitude of two D0 branes computed in this short-distance region coincides with that computed in the supergravity region $[4,7,8]$. In this case the corresponding gauge theory has sixteen supercharges, and they protect the scattering amplitude from corrections that might arise when extrapolating the length scale. Generally speaking, amplitudes that are not protected by a sufficient number of supersymmetries may behave differently in the two regions. Indeed in $[9,10]$ it is shown that the $v^{4}$-term in the D0-brane scattering amplitude in a curved space (more specifically the large volume limit of K3, called the ALE space) computed in the gauge theory region differs from the amplitude computed in the supergravity region. The former lacks subleading terms in the weak curvature expansion of the latter. Issues closely related to this were pointed out in $[11,12]$.

It is interesting to study other amplitudes in the gauge theory region and compare them with those in evaluated in the supergravity region. In [13], Polchinski and Pouliot computed membrane scattering in flat space which an exchange of a non-zero amount of M-momentum $p_{11}$. The non-zero $p_{11}$ transfer corresponds to an instanton process in the three-dimensional $N=8$ gauge theory (with sixteen supercharges) on the membrane

worldvolume. They showed that the leading term in the one-instanton calculation agrees with the leading term in the supergravity side for one unit of M-momentum exchange in the region $b \gg R_{s} \gamma$, where $b$ is the impact parameter and $\gamma=\left(1-v_{11}^{2}\right)^{-1 / 2}$ is the Lorentz boost factor for longitudinal velocity $v_{11}$. In view of the result in [10], one may suspect that such an agreement does not persist in a curved space.

In this paper, we study membrane scattering on an ALE space with non-zero $p_{11}$ transfer in the gauge theory region using the $N=4$ gauge theory (with eight superchages) in three dimensions. In curved space, there is an issue of which gauge theory should be used to describe the dynamics in the short-distance region [14-16]. In the case of membranes, the renormalizability of the corresponding three-dimensional gauge theory imposes a strong constraint. As far as we know, the only reasonable candidate is the gauge 
theory studied in [17-20] whose field content and gauge groups are arranged according to the quiver diagram. In this paper, we call this the quiver gauge theory. In the orbifold limit of the ALE space, the model can be derived from open string dynamics [17], and one can argue that turning on Fayet-Iliopoulos parameters in field theory corresponds to resolving the ALE space.

The instanton in three dimensions is a magnetic monopole. In the case studied by Polchinski and Pouliot [13], the gauge theory has $N=8$ supersymmetry, half of which is preserved by the monopole. Consequently, there are eight fermion zero modes in the instanton background, which is exactly the right number to compute the $v^{4}$-term in the membrane scattering amplitude, as the superpartner of the $F^{4}$ term in the gauge theory contains eight fermions. In our case, we find that the monopole in question breaks all the supersymmetry. Since our gauge theory has only $N=4$ supersymmetry, one can expect that the number of fermion zero-modes is still eight. We will verify that this is in fact the case. This fact helps us in estimating the normalization of the leading term in the instanton computation.

However the fact that the instanton breaks all the supersymmetry makes it difficult to construct it explicitly and evaluate its action. We address this problem in the following way. We first construct a field configuration obeying the $n$-monopole boundary condition, and show that the action for this trial field configuration is less than $n / e^{2}$ times the geodesic distance $\sigma(X, Y)$ between the membranes ( $e$ : gauge coupling constant). Since the instanton minimizes the action for the given boundary condition, the instanton action $S_{\text {instanton }}$ should clearly obey the same upper-bound. We also show that, for any field configuration with the $n$-monopole boundary condition, the action is bounded below by $n / e^{2}$ times the Euclidean distance $\|X-Y\|$ in the total field space. Thus we obtain the inequalities,

$$
\frac{n}{e^{2}}\|X-Y\|<S_{\text {instanton }}<\frac{n}{e^{2}} \sigma(X, Y),
$$

except in the orbifold limit when the inequalities above are replaced by equalities. The agreement with the supergravity computation would require the action to be exactly equal to $\frac{n}{e^{2}} \sigma(X, Y)$. Thus the gauge theory amplitude is exponentially larger the corresponding supergravity amplitude. Moreover we show that the error $\frac{n}{e^{2}} \sigma(X, Y)-S_{\text {instanton }}$ grows at least linearly in $n$ for large $n$.

The membrane scattering amplitude computed using the gauge theory is different from the supergravity result. What does this imply to the large- $N$ Matrix Theory conjecture of Banks, Fischler, Shenker and Susskind [8]? To understand the origin of the discrepancy, we examine the region of validity of the gauge theory computation within the framework 
of the large- $N$ conjecture. In Matrix Theory, the membrane arises from a collection of a large number of D0-branes [21]. The question is, in this set-up, when it is appropriate to use the three-dimensional gauge theory to describe the membrane dynamics. If the number $N$ of D0-branes is finite, there is a short-distance cutoff $\delta x \sim 1 / \sqrt{N}$ on the worldvolume of the membrane. In order for the gauge theory description to be valid, the length scale of the problem has to be larger than the cutoff length. We find that this requires that the Planck length $l_{p}$ and the distance $\sigma(X, Y)$ between the membranes be shorter than $\delta x$,

$$
l_{p}, \sigma(X, Y) \ll \delta x .
$$

Therefore, even in the large- $N$ Matrix Theory, the gauge theory description of the membrane is valid only in the short-distance regime. The gauge theory and supergravity descriptions cover complementary regimes and there is no overlap between the two. The result of this paper is an explicit example where the gauge theory computation is not applicable to the long-distance physics of membrane.

In section 2, we define the gauge theory on the membrane worldvolume and show that the instanton breaks all supersymmetries. In section 3, we prove the inequalities (1.2) and estimate the error. Section 4 is devoted to an analysis of the zero-modes in the instanton background. In section 5, we compare the gauge theory result with the supergravity prediction. The last section is devoted to a discussion on the large- $N$ Matrix Theory conjecture. In the appendix, we review how the three-dimensional gauge theory description of membranes is derived starting from a system of $N$ D0 branes, and estimate the cutoff length of the gauge theory.

\section{Quiver Gauge Theory for Membrane on ALE Space}

In Matrix Theory [8], the membrane arises from a collection of a large number of D0-branes [21]. The dynamics of the membrane is described by a supersymmetric gauge theory on the worldvolume $[22,23]$. In this section, we define the gauge theory for two membranes on the ALE space. The derivation of the action is reviewed in the appendix. We will examine the validity of this description in section 6 of this paper.

According to $[13,23]$, the coupling constant $e$ of the gauge theory is given by the string computing constant $g_{s}$ as $e^{2}=\gamma g_{s} / l_{s}$. The Lorentz boost factor $\gamma$ is given by

$$
\gamma=\frac{N / R_{s}}{L^{2} / l_{p}^{3}},
$$


where $N$ is the number of D0-branes and $L$ is the size of the membrane. In three dimensions, the gauge theory is weakly coupled if $e^{2}$ is smaller than the vacuum expectation value $|\phi|$ of the Higgs field. In this regime, the loop corrections are suppressed by powers of $e^{2} /|\phi|$, and the instanton approximation is useful. In our case, $\phi$ is typically related to the distance between the membranes $\sigma(X, Y)$ as

$$
|\phi|=\frac{\sigma(X, Y)}{l_{s}^{2}} .
$$

Therefore the gauge theory is weakly coupled when

$$
\frac{e^{2}}{|\phi|}=\frac{R_{s} \gamma}{\sigma(X, Y)} \ll 1 .
$$

In section 6, we will show that, if the gauge theory description of the membrane is valid, there is such a weakly coupled regime in the parameter space.

To simplify our notation, in this and next sections, we will set the string scale $l_{s}=1$.

\subsection{Construction of the Quiver Gauge Theory}

Let us start with a single flat membrane localized at a point in $S^{1} \times R^{3} \times \mathcal{M}$, where $\mathcal{M}$ is an ALE space of type $A_{n-1}$. According to [17], the dynamics of D0 branes propagating on an ALE space is given by a one-dimensional quiver quantum mechanics. Using this and the derivation of the membrane action from the D0 brane action in the appendix, we find that the worldvolume dynamics of the membrane is described by a three-dimensional $N=4$ supersymmetric gauge theory with gauge group $U(1)^{n}=\prod_{i=0}^{n-1} U(1)_{i}$ and one massless hypermultiplet charged $(+1,-1)$ with respect to $U(1)_{i} \times U(1)_{i+1}$ for each $i=0, \ldots, n-1$ (where $\left.U(1)_{n} \equiv U(1)_{0}\right)$. The Fayet-Iliopoulos (FI) parameter $\vec{\zeta}=\left(\vec{\zeta}_{0}, \ldots, \vec{\zeta}_{n-1}\right)$ is chosen so that $\sum_{i} \vec{\zeta}_{i}=0$. The Higgs branch is the ALE space $\mathcal{M}$ which depends on the FI parameter $\vec{\zeta}$. When $\vec{\zeta}=0$, the ALE space has an $A_{n-1}$ singularity, which is resolved for $\vec{\zeta} \neq 0$. For generic values of $\vec{\zeta}$, the moduli space consists of a single mixed Higgs-Coulomb branch $S^{1} \times R^{3} \times \mathcal{M}$, which is identified as the space transverse to the membrane.

To describe two parallel membranes, we consider the gauge theory with gauge group $U(2)^{n}=\prod_{i=0}^{n-1} U(2)_{i}$ and one hypermultiplet in $\left(2_{i}, \overline{2}_{i+1}\right)$ of $U(2)_{i} \times U(2)_{i+1}$ for each $i=0, \ldots, n-1$. The moduli space of vacua of this theory was analyzed in [20] and found to have various branches. The branch relevant in this paper is the mixed branch (called the basic branch in [20], see equation (5.51)) in which the gauge group is broken to the $U(1) \times U(1)$ subgroup of the diagonal $U(2) \subset U(2)^{n}$. This branch receives no quantum corrections and is given by the two-fold symmetric product of $S^{1} \times R^{3} \times \mathcal{M}$, which precisely 
matches the picture of two membranes at points on the transverse part of the space-time. The gauge coupling constant is equal to $e \sqrt{n}$ for all $U(2)_{i}$ so that the coupling constant for the diagonal $U(2)$ is $e$.

\subsection{Monopole and Supersymmetry}

To describe membranes moving with a large velocity in the eleventh direction we need to turn on a magnetic flux in the three-dimensional gauge theory. The momentum density $\pi_{11}$ of each membrane in the eleventh direction is given by the magnetic flux $F_{12} / 2 \pi R_{s}$ of the corresponding unbroken $U(1)$ subgroup. Therefore, in a scattering with one unit of momentum transfer, the magnetic flux $\int \mathrm{d} x^{1} \mathrm{~d} x^{2} F_{12}$ of one $U(1)$ increases by $2 \pi$ and the flux of the other $U(1)$ decreases by the same amount. This is realized as the magnetic monopole of the $S U(2)$ subgroup of the diagonal $U(2)$. Namely, this is an instanton process of this $S U(2)$.

Later we will need to know the number of fermion zero modes in this instanton background. For this, it is useful to count the number of unbroken supersymmetries. In fact, the instanton in question breaks all the supersymmetry. The easiest way to see this is to realize the gauge theory using intersecting branes [24]. The brane configuration consists of D3 and NS5-branes in type IIB theory on the flat space-time $R^{9} \times S^{1}$ which we parametrize by $x^{0}, \ldots, x^{9}$ where the $S^{1}$ is in the $x^{6}$ direction [25]. There are $n$ parallel NS5-branes spanning the $x^{0,1,2,3,4,5}$ directions and two parallel D3-branes spanning the $x^{0,1,2,6}$ directions. Since the $x^{6}$ direction is finite the worldvolume dynamics of the D3branes is at low energies described by a three-dimensional gauge theory. An analysis of the open string states shows that the theory has the same gauge symmetry and matter content as the quiver gauge theory given above.

Before we count the number of unbroken supersymmetries in the instanton background, it is useful to briefly review why this configuration has $N=4$ supersymmetry in three dimensions. Type IIB theory in flat space-time has 32 supercharges parametrized by a pair of Majorana-Weyl spinors, $\epsilon_{+}$and $\epsilon_{-}$, of the same chirality $\Gamma_{0 \ldots 9} \epsilon_{ \pm}=\epsilon_{ \pm}$. The NS5branes preserve half of the supersymmetries, namely the ones satisfying $\Gamma_{012345} \epsilon_{ \pm}= \pm \epsilon_{ \pm}$, and the D3 branes preserves those that obey $\Gamma_{0126} \epsilon_{+}=\epsilon_{-}$. Therefore $32 /(2 \cdot 2)=8$ generators are preserved, which is the number of supersymmetries of $N=4$ in three dimensions.

Now let us show that the instanton in question break all the remaining supersymmetry. As noted in [24,25], an instanton in the three-dimensional gauge theory is realized as the open Euclidean D1-brane stretched between the two D3-branes and in the $x^{6}$ direction. 


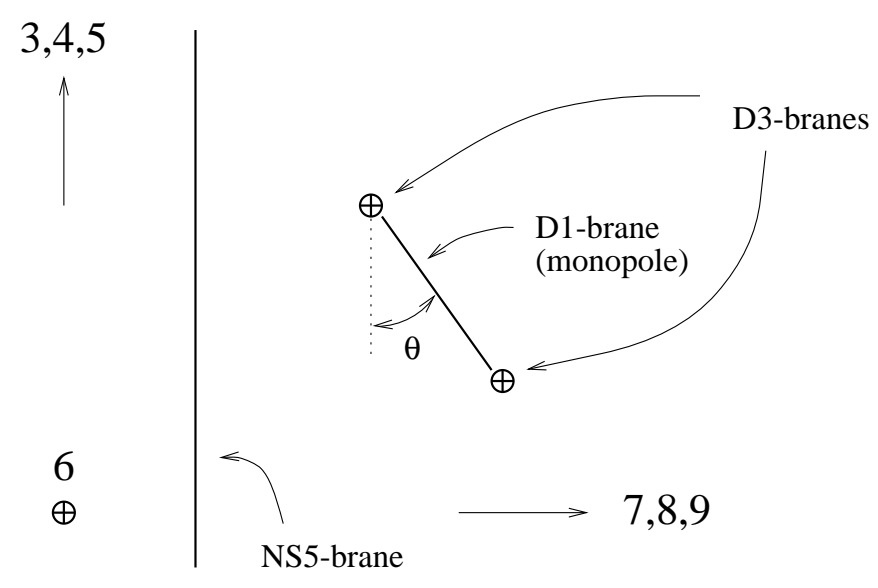

Figure 1: The monopole in the Higgs or mixed Higgs-Coulomb branch $(0<\theta<\pi)$ breaks all the supersymmetry.

If we were in the Coulomb branch, the two D3-branes would have been separated in the $x^{3,4,5}$ directions. Then the instanton realized as the D1-brane would, for D3-branes separated in $x^{3}$ only, impose the additional condition $\Gamma_{36} \epsilon_{+}=\epsilon_{-}$for unbroken supersymmetry. Since $\Gamma_{36}$ anti-commutes with $\Gamma_{012345}$, this would have been compatible with the condition $\Gamma_{012345} \epsilon_{ \pm}= \pm \epsilon_{ \pm}$associated with the NS5-branes, thus there would have been four unbroken supercharges. We are, however in the mixed Coulomb-Higgs branch. There the two D3-branes are separated in a mixed direction of $x^{3,4,5}$ and $x^{7,8,9}$, say $\cos \theta \partial_{3}+\sin \theta \partial_{7}$ (see figure 1). Thus the condition is $\left(\cos \theta \Gamma_{36}+\sin \theta \Gamma_{76}\right) \epsilon_{+}=\epsilon_{-}$. In this case, since $\Gamma_{76}$ commutes with $\Gamma_{012345}$, it is not compatible with the condition from the NS 5-branes. This proves that the supersymmetry is completely broken by instantons in the Higgs $(\theta=\pi / 2)$ or mixed branch $(\theta \neq 0, \pi / 2)$. If there were no NS5-branes, the two conditions would not have been in conflict and some of the supersymmetries would have been preserved. This corresponds to the case of $N=8$ supersymmetry discussed in [13].

This has the following consequence in the corresponding $N=2$ theories in four dimensions. Note that instantons in three dimensions are solitons in four dimensions. In four dimensions, the above observation translates into the statement that monopoles in the Higgs or mixed branches, if they exist, cannot be Bogomolny-Prasad-Sommerfeld (BPS) particles. As is well-known BPS states are in the short multiplet of $N=2$ supersymmetry consisting of four states, whereas non-BPS states are in the long multiplet consisting of sixteen states. If the monopole is not a BPS state, there must be a sufficient number of degrees of freedom to make a long multiplet. In the Higgs (or mixed) branch, one $N=2$ vector multiplets (eight states) mixes via the Higgs mechanism with one hypermultiplet 
(eight states) and in total there are the right number of states to make a long multiplet. In the Coulomb branch, one vector multiplet mixes with itself and decomposes into one BPS and one anti-BPS multiplet. In the mixed branch of $N=4$ super-Yang-Mills theory, the sixteen states mentioned above decompose again into four BPS/anti-BPS multiplets.

\section{Monopole Solution and Action}

We have found that the monopole solution in our case preserves no supersymmetry. This makes it difficult to find an exact form of the solution. We address this issue by using the following strategy.

First we start with the quiver gauge theory defined in the last section, constrain almost all massive excitations of the scalar fields to be zero and go to the low-energy effective theory. The scalar fields are subject to the D and F-term constraints and are only allowed to move along the moduli space of vacua. In this model, we construct a trial field configuration obeying the boundary condition for the $n$-monopole, and show that its action is smaller than $n / e^{2}$ times the geodesic distance $\sigma(X, Y)$ between the two membranes. Since the instanton minimizes the action, the instanton action should also obey the same upper-bound. We also show that, for any field configuration obeying this boundary condition, the action is bounded below by $n / e^{2}$ times the Euclidean distance $\|X-Y\|$ between the two membranes. Thus the instanton action for the $n$-instanton should be bounded by these two quantities as

$$
\frac{n}{e^{2}}\|X-Y\|<S_{\text {instanton }}<\frac{n}{e^{2}} \sigma(X, Y)
$$

except in the orbifold limit of the ALE space $\mathcal{M}$ when the inequalities are replaced by equalities. In the following, we assume $\mathcal{M}$ is smooth (i.e. $\vec{\zeta} \neq \overrightarrow{0}$ ) unless otherwise noted.

We then proceed by showing that the above field configuration can be lifted to the full quiver gauge theory. To solve the equations of motion of the full theory, we also have to minimize the action with respect to the massive modes. We show that this further

increases the amount of the error, $\frac{n}{e^{2}} \sigma(X, Y)-S_{\text {instanton, although the action still obeys }}$ the lower-bound in (3.1).

\subsection{Monopole Configuration in the Low Energy Theory}

The low energy bosonic degrees of freedoms of the gauge theory are the scalar fields $\left(X^{i}, Y^{i}\right)(i=1, \ldots, 7)$ taking values in the moduli space of vacua $\operatorname{Sym}^{2}\left(R^{3} \times \mathcal{M}\right)$ and 
the gauge field $a_{\mu}^{a}(a=1,2)$ of the $U(1) \times U(1)$ subgroup of the diagonal $U(2)$ and are described by the Lagrangian density,

$$
e^{2} \mathcal{L}_{e f f}=\frac{1}{4} \sum_{a=1,2}\left(\partial_{[\mu}, a_{\nu]}^{a}\right)^{2}+\frac{1}{2} g_{i j}(X) \partial_{\mu} X^{i} \partial_{\mu} X^{j}+\frac{1}{2} g_{i j}(Y) \partial_{\mu} Y^{i} \partial_{\mu} Y^{j}
$$

where $g_{i j}$ is the metric of the space $R^{3} \times \mathcal{M}$.

In order to construct the monopole, we need to retain the $\mathrm{W}$-bosons $A_{\mu}^{ \pm}$of the diagonal $U(2)$ which are generically massive on the moduli space $\operatorname{Sym}^{2}\left(R^{3} \times \mathcal{M}\right)$ but become massless at the diagonal of the symmetric product where the two membranes coincide. We therefore consider the $U(2)$ gauge theory ${ }^{1}$ with the following Lagrangian density,

$$
e^{2} \mathcal{L}_{e f f}=\frac{1}{4} \operatorname{tr} F_{\mu \nu}^{2}+\frac{1}{2} g_{i j}(X) \partial_{\mu} X^{i} \partial_{\mu} X^{j}+\frac{1}{2} g_{i j}(Y) \partial_{\mu} Y^{i} \partial_{\mu} Y^{j}+2\|X-Y\|^{2} A_{\mu}^{+} A_{\mu}^{-}
$$

where $\|X-Y\|$ is the W-boson mass given as follows. Recall that the original quiver gauge theory has three real scalar fields $\vec{\varphi}_{i}$ in the adjoint representation for each $U(2)_{i}$, and a pair of complex scalars $\left(Q_{i}, \tilde{Q}_{i}\right)$ which is in $\left(2_{i}, \overline{2}_{i+1}\right)$ for each $U(2)_{i} \times U(2)_{i+1}$. At each point of the moduli space $(X, Y)$, these matrix-valued scalars can be diagonalized $[20]$ as

$$
\vec{\varphi}_{i}=\left(\begin{array}{cc}
\vec{\varphi}(X) & 0 \\
0 & \vec{\varphi}(Y)
\end{array}\right), \quad Q_{i}=\left(\begin{array}{cc}
b_{i}(X) & 0 \\
0 & b_{i}(Y)
\end{array}\right), \quad \widetilde{Q}_{i}=\left(\begin{array}{cc}
\tilde{b}_{i}(X) & 0 \\
0 & \tilde{b}_{i}(Y)
\end{array}\right)
$$

Here both $\left(b_{i}(X), \tilde{b}_{i}(X)\right)$ and $\left(b_{i}(Y), \tilde{b}_{i}(Y)\right)$ obey the D and F-term constraints of the $\prod_{i} U(1)_{i}$ gauge theory for a single membrane located at $X$ and $Y$ respectively. The Euclidean distance $\|X-Y\|^{2}$ is then defined as

$$
\|X-Y\|^{2} \equiv n|\vec{\varphi}(X)-\vec{\varphi}(Y)|^{2}+\sum_{i}\left(\left|b_{i}(X)-b_{i}(Y)\right|^{2}+\left|\tilde{b}_{i}(X)-\tilde{b}_{i}(Y)\right|^{2}\right)
$$

In other words, $\|X-Y\|$ is the Euclidean distance of some representative points of $X$ and $Y$ in the vector space for the scalar fields of the $\prod_{i} U(1)_{i}$ gauge theory for a single

\footnotetext{
${ }^{1}$ The action in $(3.3)$ is actually the $U(2)$ gauge theory in a specific unitary gauge, namely one where all scalar fields are diagonal. (In the quiver gauge theory, all scalar fields transform in the adjoint of the diagonal $U(2))$. By introducing an additional 'compensating' $U(2)$ valued field one can make (3.3) into a gauge theory where the gauge has not yet been fixed. The unitary gauge chosen here is equivalent to choosing the gauge $\phi=$ diagonal in the usual BPS equation $F=* D \phi$. For the BPS monopole solution, this gauge cannot be chosen globally, but only on the two hemispheres of a sphere at infinity. Along the equator, one has to glue the solutions together using a non-trivial transition function. In principle we would have to do the same thing for the trial monopole configuration that we will construct, but it is easy to see that exactly the same transition functions can be used to promote our trial configuration into an everywhere well-defined configuration.
} 
membrane. That the W-boson mass of the low energy theory is equal to the Euclidean distance in the total field space of the quiver gauge theory was pointed out in [9]. The value of the Euclidean distance clearly depends on how to take the representative points (3.4). Below we will specify how it is done in our case.

Let us first rewrite the action (3.3). For a function $f(X, Y)$ on $\operatorname{Sym}^{2}\left(R^{3} \times \mathcal{M}\right)$, define

$$
\Lambda=\left(\begin{array}{cc}
f(X, Y) & 0 \\
0 & -f(X, Y)
\end{array}\right) .
$$

We can use this to recombine the kinetic term for the gauge field as

$$
\operatorname{tr} F_{\mu \nu}^{2}=\operatorname{tr}\left(F_{\mu \nu}-\epsilon_{\mu \nu \rho} D_{\rho} \Lambda\right)^{2}+2 d \operatorname{tr} F \Lambda-2\left(\partial_{\mu} f\right)^{2}-8 f(X, Y)^{2} A_{\mu}^{+} A_{\mu}^{-} .
$$

The effective Lagrangian density (3.3) then becomes

$$
\begin{aligned}
e^{2} \mathcal{L}_{e f f}= & \frac{1}{4} \operatorname{tr}\left(F_{\mu \nu}-\epsilon_{\mu \nu \rho} D_{\rho} \Lambda\right)^{2}+\frac{1}{2}\left(\partial_{\mu} X^{i}, \partial_{\mu} Y^{i}\right) M_{i j}\left(\begin{array}{c}
\partial_{\mu} X^{j} \\
\partial_{\mu} Y^{j}
\end{array}\right)+ \\
& +\frac{1}{2} d \operatorname{tr} F \Lambda+2\left[\|X-Y\|^{2}-f(X, Y)^{2}\right] A_{\mu}^{+} A_{\mu}^{-} .
\end{aligned}
$$

where the matrix $M_{i j}$ is given by

$$
M_{i j}=\left(\begin{array}{cc}
g_{i j}(X)-\frac{1}{2} \frac{\partial f}{\partial X^{i}} \frac{\partial f}{\partial X^{j}} & -\frac{1}{2} \frac{\partial f}{\partial X^{i}} \frac{\partial f}{\partial Y^{j}} \\
-\frac{1}{2} \frac{\partial f}{\partial Y^{i}} \frac{\partial f}{\partial X^{j}} & g_{i j}(Y)-\frac{1}{2} \frac{\partial f}{\partial Y^{i}} \frac{\partial f}{\partial Y^{j}}
\end{array}\right) .
$$

We now construct a field configuration which sets the first two terms in the Lagrangian density (3.8) to be zero. This can be done by making use of the standard BPS monopole solution to the $S U(2)$ gauge theory with a single adjoint scalar field $\Phi(x)$. The BPS equation for them is

$$
F_{\mu \nu}=\epsilon_{\mu \nu \rho} D_{\rho} \Phi
$$

A general $n$-monopole solution to (3.10) is given by Corrigan and Goddard [26]. By choosing our $U(2)$ gauge field $A_{\mu}$ to be equal the $n$-monopole solution to (3.10) and by requiring the massless scalars $(X, Y)$ to obey

$$
f(X(x), Y(x))=\phi(x), \quad( \pm \phi: \text { eigenvalues of } \Phi)
$$

we can set the first term in the Lagrangian density (3.8) to be zero.

So far the function $f(X, Y)$ has been arbitrary. To obtain the upper bound in (3.1) we choose the function to be the geodesic distance between $X$ and $Y$,

$$
f(X, Y)=\sigma(X, Y) .
$$


With this choice, the matrix $M_{i j}$ is positive semi-definite. This follows from the HamiltonJacobi equation for the geodesic distance

$$
g^{i j}(X) \frac{\partial \sigma}{\partial X^{i}} \frac{\partial \sigma}{\partial X^{j}}+g^{i j}(Y) \frac{\partial \sigma}{\partial Y^{i}} \frac{\partial \sigma}{\partial Y^{j}}=1 .
$$

The second term in the Lagrangian density (3.8) can be set to be zero if $\left(\partial_{\mu} X^{i}, \partial_{\mu} Y^{i}\right)$ is the zero eigenvector of the matrix $M_{i j}$, namely

$$
\begin{aligned}
g_{i j}(X) \partial_{\mu} X^{j} & =\frac{\partial \sigma}{\partial X^{i}} \partial_{\mu} \sigma(X(x), Y(x)) \\
g_{i j}(Y) \partial_{\mu} Y^{j} & =\frac{\partial \sigma}{\partial Y^{i}} \partial_{\mu} \sigma(X(x), Y(x)) .
\end{aligned}
$$

To solve this equation, we use the following property of geodesics. Suppose $\left(X_{0}(\tau), Y_{0}(\tau)\right)$ is a geodesics on $\operatorname{Sym}^{2}\left(R^{3} \times \mathcal{M}\right)$ with $\tau$ being the proper time and with the initial condition

$$
X_{0}=Y_{0} \quad \text { at } \tau=0 .
$$

Because of the property of geodesics,

$$
g_{i j}\left(X_{0}\right) \frac{d X_{0}^{j}}{d \tau}=\frac{\partial \sigma\left(X_{0}, Y_{0}\right)}{\partial X_{0}^{i}}, \quad g_{i j}\left(Y_{0}\right) \frac{d Y_{0}^{j}}{d \tau}=\frac{\partial \sigma\left(X_{0}, Y_{0}\right)}{\partial Y_{0}^{i}} .
$$

the equations (3.11) and (3.14) are both satisfied by setting

$$
X(x)=X_{0}(\phi(x)), \quad Y(x)=Y_{0}(\phi(x)) .
$$

Thus we find that, for the field configuration given by (3.10) and (3.17), the Lagrangian density (3.8) becomes

$$
e^{2} \mathcal{L}_{e f f}=\frac{1}{2} d \operatorname{tr} F \Lambda+2\left[\|X-Y\|^{2}-\sigma(X, Y)^{2}\right] A_{\mu}^{+} A_{\mu}^{-} .
$$

The second term in the right-hand side is negative definite for the following reason. As we will show in the next subsection, in order to lift the above monopole solution to the original quiver gauge theory, we must choose a gauge slice for the $\prod_{i} U(2)_{i}$ gauge symmetry that is normal to the gauge variation at any value of $\tau$. For such a choice, the hyper-Kähler quotient metric induced on the geodesic is the same as the restriction of the flat Euclidean metric to the gauge slice, and the geodesic length $\sigma(X, Y)$ is the same as the length of the slice measured by the flat metric. Since $\|X-Y\|$ is the length of the straight segment connecting the same end points measured by the same flat metric, this must be smaller than the length of the slice which is equal to $\sigma(X, Y)$. Since the first term of (3.18) is a total derivative, its integral is determined by the $n$-monopole boundary condition. Thus 
we find that the action for the field configuration (3.10), (3.17) is bounded above by the geodesic distance as

$$
S_{\text {eff }}=\int d^{3} x \mathcal{L}_{e f f}<\frac{n}{e^{2}} \sigma(X, Y)_{\mid x \rightarrow \infty}
$$

Since the instanton minimizes the action, $S_{\text {instanton }}$ is smaller than the action $S_{\text {eff }}$ for the trial monopole configuration considered here. Thus it should also obey the same upper bound.

We can also use (3.8) to find a lower bound on the instanton action. For this, we set $f(X, Y)$ to be the Euclidean distance $\|X-Y\|$. The Lagrangian density then becomes

$$
e^{2} \mathcal{L}_{e f f}=\frac{1}{4} \operatorname{tr}\left(F_{\mu \nu}-\epsilon_{\mu \nu \rho} D_{\rho} \Lambda\right)^{2}+\frac{1}{2}\left(\partial_{\mu} X^{i}, \partial_{\mu} Y^{i}\right) M_{i j}\left(\begin{array}{c}
\partial_{\mu} X^{j} \\
\partial_{\mu} Y^{j}
\end{array}\right)+\frac{1}{2} d \operatorname{tr} F \Lambda .
$$

It is easy to see that the matrix $M_{i j}$ is now strictly positive definite and the second term in the right-hand side is positive. We thus obtain the lower bound

$$
\frac{n}{e^{2}}\|X-Y\|<S_{e f f}
$$

for any field configuration satisfying the $n$-monopole boundary condition.

\subsection{Estimate of the Error}

Let us estimate the size of the error,

$$
\frac{n}{e^{2}} \sigma(X, Y)-S_{e f f}=\frac{2}{e^{2}} \int d^{3} x\left[\sigma(X(x), Y(x))^{2}-\|X(x)-Y(x)\|^{2}\right] A_{\mu}^{+} A_{\mu}^{-} .
$$

When $n=1$, the main contribution to the error comes from the region where the distance $r$ from the center of monopole is less than the Compton wavelength of the W-boson. When the distance between $X$ and $Y$ is shorter than the typical curvature radius $R_{c}$ of $\mathcal{M}$, the difference between the geodesic distance and the Euclidean distance is estimated as

$$
\sigma(X, Y)^{2}-\|X-Y\|^{2} \sim \frac{\sigma^{4}(X, Y)}{R_{c}^{2}} .
$$

In our monopole configuration,

$$
\begin{aligned}
\sigma(X(r), Y(r)) & =\phi(r) \\
& =\sigma \operatorname{coth}(\sigma r)-\frac{1}{r}
\end{aligned}
$$

where $\sigma$ in the right-hand side is the value of $\sigma(X, Y)$ at $r=\infty$. By combining with the known expression for the $\mathrm{W}$-bosons $A^{ \pm}$in the BPS solution, we find

$$
\left[\sigma^{2}(X, Y)-\|X-Y\|^{2}\right] A_{\mu}^{+} A_{\mu}^{-} \sim \frac{\sigma^{6}}{R_{c}^{2}} F(\sigma r)
$$


where $F(\xi)$ is a certain function which decays exponentially for large $\xi$. The error (3.22) for $n=1$ is then estimated as

$$
\frac{1}{e^{2}} \sigma(X, Y)-S_{e f f} \sim \frac{\sigma^{3}}{e^{2} R_{c}^{2}} \int_{0}^{\infty} d \xi \xi^{2} F(\xi) .
$$

This in fact is of the same order as the difference of the geodesic distance and the Euclidean distance between $X$ and $Y$. Because of the exponential suppression by $F(\sigma r)$, the main contribution to the integral (3.22) is from the region within the Compton wavelength of the W-boson. Since the instanton action $S_{\text {instanton }}$ is in general smaller than the trial monopole configuration, the actual error $\frac{1}{e^{2}} \sigma-S_{\text {instanton }}$ for the instanton action can be larger than the one given by (3.26).

Now let us estimate the magnitude of the error for an arbitrary value of $n$. For this, it is easiest to consider the case of the well-separated BPS monopoles as our trial configuration. If the distance between monopoles is larger than the Compton wavelength of the W-boson, the behavior of the $n$-monopole solution near the core of each monopole can be approximated by that of the single monopole solution. Since the integrand of (3.22) is positive definite, there is no possibility of cancellation of the errors. Thus for the well-separated $n$-monopole,

$$
\frac{n}{e^{2}} \sigma(X, Y)-S_{e f f} \sim \frac{n \sigma^{3}}{e^{2} R_{c}^{2}} \int_{0}^{1} d \xi \xi^{2} F(\xi) .
$$

Since the instanton action is smaller than that of the trial configuration, the actual error can be larger than this. Thus we find that the difference between the geodesic distance $\frac{n}{e^{2}} \sigma(X, Y)$ and the instanton action $S_{\text {instanton }}$ is at least of the order $n$ for large $n$.

\subsection{Lifting to the Quiver Gauge Theory}

The low-energy theory with the Lagrangian density (3.3) is not renormalizable in three dimensions. It could still be used for computations if the energy scale of the problem is smaller than that set by the curvature of the moduli space $\mathcal{M}$. The energy scale for the instanton solution is determined by how fast the fields $A_{\mu}, X$ and $Y$ change on the membrane worldvolume and it is controlled by the values of $X$ and $Y$ at the infinity, typically by the distance $\sigma(X, Y)$ between the membranes. Thus we expect that computations done in the low-energy theory (3.3) suffer large corrections when the distance $\sigma(X, Y)$ is comparable to the curvature radius of $\mathcal{M}$. In particular, for the

purpose of computing subleading terms in the weak curvature expansion of the scattering 
amplitudes, the analysis using the low-energy theory is not sufficient and we have to use the renormalizable gauge theory discussed in section 2 .

It turns out that the instanton action for the full quiver gauge theory obeys the same bound (3.1) as the instanton action for the low-energy effective theory. In this subsection, we show that the field configuration constructed in the previous subsection can be lifted to that of the quiver gauge theory and that the value of the action for the lifted configuration remains the same. Thus the upper-bound for the instanton action given by (3.1) also holds for the quiver gauge theory. In the next subsection, we show that the instanton action for the quiver gauge theory is in fact lower than that of the low energy theory, but is still bounded from below as in (3.1).

First let us briefly review the procedure to obtain the non-linear sigma model on the Kähler quotient as the classical low-energy effective theory of the quiver gauge theory. (The sigma model on the hyper-Kähler quotient can be obtained simply by restricting to the zero of the F-term potential.) Suppose we are given a complex scalar field $\Phi$ in a representation of the gauge group $G$, with the Lagrangian density

$$
\mathcal{L}=\frac{1}{2 e^{2}}\left\|F_{\mu \nu}\right\|^{2}+\left\|D_{\mu} \Phi\right\|^{2}+\frac{e^{2}}{2} \sum_{a=1}^{\operatorname{dim} G}\left|\Phi^{\dagger} T_{a} \Phi-\zeta_{a}\right|^{2} .
$$

The last term is the D-term potential where $T_{a}$ 's are the generators of the gauge group and $\zeta_{a}$ is the FI parameter which has values in the center of the gauge group only. We consider for simplicity only the region of the values of the scalar field where the gauge group is completely broken. The gauge field acquires a mass from the second term and we can first integrate out the gauge field. The second term $\|D \Phi\|^{2}$ contains terms linear and quadratic in the gauge field and we can eliminate the gauge field by completing the square, ignoring their kinetic terms for the moment. The variation of this term with respect to the gauge field is expressed, with the aid of the D-term constraint, as $\delta\|D \Phi\|^{2}=-2 \delta A_{\mu}^{a} \Phi^{\dagger} T_{a} D_{\mu} \Phi$. It is extremized if

$$
M_{\bar{a} b} A_{\mu}^{b}=-\left(T_{a} \Phi\right)^{\dagger} \partial_{\mu} \Phi
$$

where $M_{\bar{a} b}=\left(T_{a} \Phi\right)^{\dagger} T_{b} \Phi$ is a matrix which has an inverse $M^{b \bar{a}}$ if the gauge group is completely broken by $\Phi$. Next, we solve (3.29) with respect to $A_{\mu}^{b}$ and plug the solution into $\|D \Phi\|^{2}$. We obtain

$$
\left.\|D \Phi\|^{2}\right|_{(3.29)}=\partial_{\mu} \Phi^{\dagger} \partial^{\mu} \Phi-\left[\partial_{\mu} \Phi^{\dagger} T_{b} \Phi\right] M^{b \bar{a}}\left[\left(T_{a} \Phi\right)^{\dagger} \partial_{\mu} \Phi\right] .
$$

This yields the non-linear sigma model Lagrangian density $G_{i \bar{\jmath}} \partial_{\mu} X^{i} \partial_{\mu} X^{\bar{\jmath}}$ on the Kähler quotient for an arbitrary choice of the gauge slice $X \rightarrow \Phi(X)$ in the D-constrained manifold. 
Substituting (3.29) into the gauge kinetic term $\left\|F_{\mu \nu}\right\|^{2}$, we obtain a higher derivative term for $\Phi$. Therefore the resulting Lagrangian density would be in general different from the one given by (3.3). Fortunately this is not the case for the monopole solution constructed in the previous subsection. If we can choose a gauge slice $\Phi(X)$ so that it is everywhere normal to the gauge orbit, the right-hand side of (3.29) vanishes. In general, such a slice may or may not exist. If it exists, the term in $\|D \Phi\|^{2}$ linear in $A_{\mu}$ vanishes for such a gauge slice and the second term of (3.28) becomes of the form

$$
\|D \Phi\|^{2}=G_{i \bar{\jmath}} \partial_{\mu} X^{i} \partial_{\mu} X^{\bar{\jmath}}+\left\|A_{\mu} \Phi(X)\right\|^{2} .
$$

Therefore we can set the gauge field $A_{\mu}$ to be equal to zero and still get the non-linear sigma-model Lagrangian density.

In our quiver gauge theory, such a normal slice does not exist for a generic configuration $(X(x), Y(y))$. Fortunately our monopole solution is special in the sense that it evolves along a one-dimensional subspace in the total field space. This is because $(X, Y)$ depends on $x^{\mu}$ only through the single function $\phi(x)$ as in (3.17). Therefore we can choose a normal slice for this configuration and obtain (3.31) for the kinetic term of the scalar field. The normal section can be chosen so that all bifundamentals are simultaneously diagonalized as in (3.5). If we keep only the gauge field of the diagonal $U(2)$ subgroup, then the term quadratic in $A_{\mu}$ in $(3.31)$ is

$$
\left\|A_{\mu} \Phi(X, Y)\right\|^{2}=\|X-Y\|^{2} A_{\mu}^{+} A_{\mu}^{-},
$$

where $\|X-Y\|^{2}$ is defined by (3.5). Thus, the Lagrangian density of the quiver gauge theory for this configuration is the same as $\mathcal{L}_{\text {eff }}$ in (3.3) if we set all the massive fields equal to zero, except for the $\mathrm{W}$-bosons in the diagonal $U(2)$ subgroup.

\subsection{Effects of Massive Modes}

We have shown that there is a particular configuration of the quiver gauge theory, satisfying the boundary condition for the $n$-monopole, such that its action is bounded from above by $\frac{n}{e^{2}} \sigma(X, Y)$. The configuration, however, may not be a solution to the full equations of motion of the quiver theory as we are simply setting the massive fields to be equal to zero, except for the W-boson of the diagonal $U(2)$. In this subsection, we will estimate the effects of the massive modes. We will show that they increase the amount of the error estimated in section 3.2.

When we turn on the massive modes of the scalar fields, the monopole solution will deviate from the BPS configuration (3.10), (3.17). The massive modes are not negligible 
when the moduli space embedded in the large field space has non-zero extrinsic curvature. In such a case, the motion of the massless modes along the moduli space in general generates a centrifugal force (or rather centripetal force in this case as we are considering an Euclidean rather and Minkowskian equation of motion), which will cause the trajectory to deviate from the moduli space. The situation is illustrated in figure 2. Here we will show that, unless the FI parameters $\vec{\zeta}$ are all equal to zero, the centripetal force is nonzero. The case with $\vec{\zeta}=0$ corresponds to the orbifold limit of $\mathcal{M}$. Thus, with the massive fields turned on, the monopole solution deviates from the BPS configuration found in the previous section. This is an additional difficulty in finding an exact form of the instanton solution. However, the very fact that the actual solution deviates from the BPS solution means that the action for the solution is smaller than the one evaluated in the last two subsections.

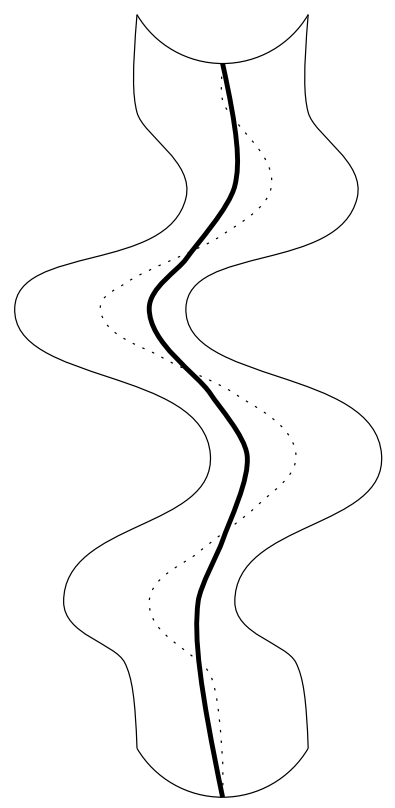

Figure 2: The trajectory does not follow the ground state of the potential due to the centripetal force.

Let us study how the solution deviates from the sigma-model solution. In the gauge theory described in section 2.1, there is one hypermultiplet field $\left(Q_{i}, \tilde{Q}_{i}\right)$ which is in $\left(2_{i}, \overline{2}_{i}\right)$ for each $U(2)_{i} \times U(2)_{i+1}$. The $\mathrm{D}$ and F-terms for each $U(2)_{i}$ are

$$
D_{i}^{A}=\sigma_{a b}^{A}\left(\Phi_{i-1}^{\dagger a} \Phi_{i-1}^{b}-\bar{\Phi}_{i}^{a} \Phi_{i}^{\dagger b}\right)=\zeta_{i}^{A} \mathbf{1}_{i},
$$


where $a, b=1,2$ are the hyperkähler indices as

$$
\left(\Phi_{i}^{1}, \Phi_{i}^{2}\right)=\left(Q_{i}, \tilde{Q}_{i}^{*}\right),
$$

and $\sigma^{A=1,2,3}$ are the standard Pauli matrices. The potential for the scalar fields of the gauge theory is then

$$
V(\Phi)=\sum_{i} \sum_{A=1}^{3} \operatorname{tr}\left|D_{i}^{A}-\zeta_{i}^{A} \mathbf{1}_{i}\right|^{2},
$$

where tr is over the $U(2)_{i}$ gauge group representation.

Suppose the general solution to $V(\Phi)=0$ is parametrized by moduli fields $\phi^{\alpha}$ as $\Phi_{i}^{a}=\Phi_{i(0)}^{a}(\phi)$. By construction, the BPS configuration satisfies

$$
\left(\frac{\partial D_{i}^{A}}{\partial \Phi_{i}^{a}}\right)_{\mid \Phi=\Phi_{0}} \frac{\partial \Phi_{i(0)}^{a}}{\partial \phi^{\alpha}}=0,
$$

namely normal vectors to the moduli space are given by derivatives of the D-terms $D_{i}^{A}$ with respect to $\Phi$, modulo gauge transformation. As the metric in the total field space is flat, the centripetal force is given by

$$
\begin{aligned}
F_{i}^{A} & =\left(\frac{\partial D_{i}^{A}}{\partial \Phi_{i}^{a}}\right)_{\mid \Phi=\Phi_{0}} \frac{\partial^{2} \Phi_{i(0)}^{a}}{\partial \phi^{\alpha} \partial \phi^{\beta}} D_{\mu} \phi^{\alpha} D^{\mu} \phi^{\beta} \\
& =\sigma_{a b}^{A}\left(D_{\mu} \Phi_{(0) i-1}^{\dagger a} D^{\mu} \Phi_{(0) i-1}^{b}-D_{\mu} \bar{\Phi}_{(0) i}^{a} D^{\mu} \Phi_{(0) i}^{\dagger b}\right) .
\end{aligned}
$$

Here we used (3.36) a couple of times to reduce the equation.

Now we are ready to show that the centripetal force $F_{i}^{A}$ is in fact non-zero for the solution constructed in the previous section, except in the orbifold limit of $\mathcal{M}$. As the fields $\Phi$ in the monopole configuration depend on $x$ through the propertime $\tau=\phi(x)$, the vanishing of the force $F_{i}^{a}=0$ would require

$$
\sigma_{a b}^{A}\left(D_{\tau} \Phi_{(0) i-1}^{\dagger a} D_{\tau} \Phi_{(0) i-1}^{b}-D_{\tau} \bar{\Phi}_{(0) i}^{a} D_{\tau} \Phi_{(0) i}^{\dagger b}\right)=0 .
$$

This, however, is the same as the D and F-term constraints for $\operatorname{Sym}^{2}\left(R^{3} \times \mathcal{M}\right)$ with $\vec{\zeta}=0$. This means that $D_{r} \Phi_{(0)}$ is on the orbifold. It is straightforward to show that this is possible only if $\Phi_{(0)}$ itself is on the orbifold. This means $\vec{\zeta}=0$. Thus we find that the centripetal force is non-zero unless $\vec{\zeta}=0$.

On the other hand, the action is clearly larger than the Euclidean distance in the total field space. To see this, suppose we remove the D an F-term potentials from the Lagrangian density. We then have the $\prod_{i} U(2)_{i}$ gauge theory coupled to massless scalar fields. By using the standard BPS argument, one can easily show that the action for 
the field configuration obeying the $n$-monopole boundary condition is bounded below by $\frac{n}{e^{2}}\|X-Y\|$. Adding the $\mathrm{D}$ and F-term potentials back would simply increase the value of the action. Thus the action of the quiver gauge theory is also bounded below by the Euclidean distance.

This concludes the proof of the inequality

$$
\frac{n}{e^{2}}\|X-Y\|^{2}<S_{\text {instanton }}<\frac{n}{e^{2}} \sigma(X, Y),
$$

and the estimate of the error $\frac{n}{e^{2}} \sigma(X, Y)-S_{\text {instanton. }}$.

\section{Instanton Calculation}

In this section we briefly summarize some aspects of the instanton calculation that one needs to do in order to compute the leading term in the membrane scattering process. We will work in the orbifold limit where all F-I parameters vanish. In this limit we we will see that there are precisely eight fermionic zero modes, all originating in the broken supersymmetries. Turning on the FI parameters will make the calculation quite a bit more involved, but it is clear that for sufficiently small parameters the number of fermionic zeromodes will remain eight and that the resulting amplitude will be nonvanishing.

To perform the instanton calculation we adapt the notation of [13] in order to facilitate a comparison with the $N=8$ calculation done in that paper. Thus, we start with $N=1$ supersymmetric Yang-Mills theory in ten dimensions, and dimensionally reduce the theory to three dimensions. This yields an $N=8$ theory in three dimensions, which can also be viewed as an $N=4$ theory with an extra adjoint hypermultiplet. We can then change the hypermultiplet representation from adjoint to some arbitrary other representation while preserving half of the supersymmetry.

The ten-dimensional indices will be denoted by capitals $M=1, \ldots, 10$, and we will decompose this into a six dimensional index $m=1, \ldots, 6$ and an index $\alpha=7, \ldots, 10$. The six dimensional index can be further decomposed into the three-dimensional space-time index $\mu=1,2,3$ and another index $p=4,5,6$. The ten-dimensional gamma matrices are $\gamma_{\mu}=\sigma_{\mu} \otimes 1 \otimes \tau_{1}, \gamma_{p}=1 \otimes \Gamma_{p} \otimes \tau_{2}$, and $\gamma_{\alpha}=1 \otimes \Gamma_{\alpha} \otimes \tau_{2}$. Here, $\sigma$ and $\tau$ are Pauli matrices and $\Gamma$ is a set of $8 \times 8$ seven-dimensional gamma-matrices. One finds that $\gamma_{11}=1 \otimes 1 \otimes \tau_{3}$ and that the charge conjugation matrix is $C=\sigma_{2} \otimes C_{(7)} \otimes \tau_{1}$. The ten-dimensional fermion and the supersymmetry parameter $\epsilon$ are both Majorana-Weyl. The reduction from ten to three dimensions is performed by decomposing the ten-dimensional index as indicated above. The $4, \ldots, 10$ components of the gauge field become scalars in three dimensions. 
$\varphi_{p} \equiv A_{p}$ are three scalar fields in the adjoint and part of the vector multiplet, whereas $Q_{\alpha} \equiv A_{\alpha}$ are the scalars in the hypermultiplet ${ }^{1}$. The fermion is decomposed in the + and - eigenvalues of the operator

$$
P=\gamma_{7} \gamma_{8} \gamma_{9} \gamma_{10}
$$

The + eigenvalues are the adjoint fermions in the vector multiplet denoted by $\lambda$, whereas the - eigenvalues are the fermions in the hypermultiplet denoted by $\psi$. The supersymmetries that survive are those that satisfy $P \epsilon=\epsilon$. The action of the three-dimensional $N=4$ theory reads

$$
S=\frac{1}{e^{2}} \int d^{3} x\left(\frac{1}{4}\left(F_{m n} F_{m n}+2 F_{m \alpha} F_{m \alpha}+D_{\alpha \beta} D_{\alpha \beta}\right)+\frac{i}{2}(\bar{\lambda}, \bar{\psi}) \gamma_{M} D_{M}(\lambda, \psi)\right) .
$$

Here $F_{\mu \alpha}=D_{\mu} Q_{\alpha}$, etc. The quantity $D_{\alpha \beta}$ contains the D- and F-term equations, to turn on a FI parameter one just has to add suitable constants to $D_{\alpha \beta}$.

In the orbifold limit, a BPS monopole has the property that the gauge field $A_{\mu}^{i}$ is independent of $i$, i.e. only a gauge field for the diagonal $U(2)$ is turned on, and that $Q_{\mu}^{i}$, the hypermultiplet transforming as $\left(2_{i}, \overline{2}_{i+1}\right)$, is also independent $i$. This is precisely a normal slice as discussed in section 3.3. The BPS equations read

$$
F_{\mu p}^{i}=\frac{1}{2} n_{p} \epsilon_{\mu \nu \rho} F_{\mu \nu}^{i}=n_{p} B_{\mu}, \quad F_{\mu \alpha}^{i}=\frac{1}{2} n_{\alpha} \epsilon_{\mu \nu \rho} F_{\mu \nu}^{i}=n_{\alpha} B_{\mu}
$$

where $B_{\mu}=\frac{1}{2} \epsilon_{\mu \nu \rho} F_{\mu \nu}^{i}$ is the magnetic field and $n_{p} n_{p}+n_{\alpha} n_{\alpha}=1$ are the components of a unit vector.

The fermion zero modes can be obtained from the supersymmetry variations of $\lambda$ and $\psi$

$$
\begin{aligned}
\delta \lambda & =\frac{i}{2} F_{m n} \gamma_{m} \gamma_{n} \epsilon+\frac{i}{2} D_{\alpha \beta} \gamma_{\alpha} \gamma_{\beta} \epsilon \\
\delta \psi & =\frac{i}{2} F_{m \alpha} \gamma_{[m} \gamma_{\alpha]} \epsilon .
\end{aligned}
$$

evaluated in a monopole background. This yields the zero modes

$$
\left(\begin{array}{c}
\lambda \\
\psi
\end{array}\right)=\left(\begin{array}{c}
-B_{\mu}\left(\sigma_{\mu} \otimes\left(1+n_{p} \Gamma_{p}\right) \otimes 1\right) \epsilon \\
-B_{\mu}\left(\sigma_{\mu} \otimes\left(n_{\alpha} \Gamma_{\alpha}\right) \otimes 1\right) \epsilon
\end{array}\right) .
$$

These are somewhat different from the ones in [13], because the unbroken supersymmetries do not depend on the choice of monopole.

\footnotetext{
${ }^{1}$ Since the hypermultiplets can be in a complex representation, one should in fact first combine $Q_{7}, Q_{8}$ and $Q_{9}, Q_{10}$ into two complex adjoint scalars and then replace the adjoint index by some other representation
} 
To determine the bosonic zero modes, we need to impose a suitable gauge. If $D_{m}, D_{\alpha}$ are the background covariant derivatives, i.e. $\left(D_{\alpha} Q_{\beta}\right)^{a}=\left(Q_{\alpha} T^{a} Q_{\beta}\right)$, then we impose

$$
D_{m} a_{m}+D_{\alpha} q_{\alpha}=0
$$

where $a_{m}$ and $q_{\alpha}$ are quantum fluctuations around $A_{m}$ and $Q_{\alpha}$. Then it is easy to find the three translational zero modes indexed by $\nu=1,2,3$

$$
a_{m}=F_{\nu m}, \quad q_{\alpha}=F_{\nu \alpha} .
$$

The fourth zero mode is associated to $U(1)$ rotations. As in [13] or in [27] one finds that it reads

$$
a_{\mu}=\frac{n_{p} F_{\mu p}+n_{\alpha} F_{\mu \alpha}}{\|X-Y\|}, \quad a_{p}=q_{\alpha}=0
$$

The denominator is the Euclidean distance in field space as before.

The inner products of these zero modes form a diagonal four by four matrix, as follows from the fact that $\int d^{3} x\left(F_{\mu \rho} F_{\nu \rho}+B_{\mu} B_{\nu}\right)=\delta_{\mu \nu} \int B_{\rho} B_{\rho}$, and $\int d^{3} x\left(F_{\mu \nu} B_{\mu}\right)=0$.

Next, we consider the one-loop determinants. The gauge fixing term is $\sim \int d^{3} x\left(\left(D_{m} a_{m}\right)^{2}+\right.$ $\left.\left(D_{\alpha} q_{\alpha}\right)^{2}\right)$, and the corresponding ghost term in the Lagrangian, $b D^{2} c$, gives rise to $\operatorname{det}\left(-D^{2}\right)^{k}$ in the one-loop path integral for the $U(2)^{k}$ gauge theory. The bosonic fluctuations add up to

$$
a_{m} \Delta_{m n} a_{n}+a_{m} \Delta_{m \alpha} q_{\alpha}+q_{\alpha} \Delta_{\alpha m} a_{m}+q_{\alpha} \Delta_{\alpha \beta} q_{\beta}
$$

where $\Delta_{m n}=-D^{2} \delta_{m n}-2 F_{m n}$ and similarly for the other components of $\Delta$.

In order to simplify the form of the kinetic term we try to diagonalize $F$ as much as possible. Since the quiver gauge theory has a cyclic $\mathbf{Z}_{k}$ symmetry it is convenient to perform a discrete Fourier transformation and replace $a_{m}^{i}, q_{\alpha}^{i}$ by $a_{m}^{r}, q_{\alpha}^{r}$, where $r$ is the Fourier label $r=0, \ldots, k-1$. One has to be a bit careful as one is dealing with real fields, but the result is that the bosonic kinetic term contains $6 k$ copies of the operator $-D^{2}$ and for each $r$ one copy of the four-component operator

$$
\Delta_{r}=-D^{2}-2 \operatorname{Tr}\left(a_{\mu}^{r}\left[F_{\mu \nu}, a_{\nu}^{r}\right]\right)-2 \operatorname{Tr}\left(a_{\mu}^{r}\left[B_{\mu}, \lambda_{r} X^{r}\right]\right)+2 \operatorname{Tr}\left(\lambda_{r} X^{r}\left[B_{m u}, a_{\mu}^{r}\right]\right)
$$

acting on the four components $\left(a_{\mu}^{r}, X^{r}\right)$. Here, we defined

$$
\begin{aligned}
X^{r} & =\frac{1}{\lambda_{r}}\left(n_{p} a_{p}^{r}+\frac{1+\cos (2 \pi r / k)}{2} n_{\alpha} q_{\alpha}^{r}+\frac{\sin (2 \pi r / k)}{2} n_{\alpha} q_{\alpha}^{-r}\right) \\
\lambda_{r} & =\sqrt{\left|n_{p}\right|^{2}+\cos ^{2}(r \pi / k)\left|n_{\alpha}\right|^{2}} .
\end{aligned}
$$


After a further change of basis $\Delta_{r}$ can be rewritten as

$$
\Delta_{r}=-D^{2}-i\left(1+\lambda_{r}\right) B_{\mu}\left(\sigma_{\mu} \otimes 1\right)-i\left(1-\lambda_{r}\right) B_{\mu}\left(1 \otimes \sigma_{\mu}\right) .
$$

The square of the Dirac operators that appear in the fermion kinetic terms (one has to treat $\psi$ and $\lambda$ together) is found to be equal to

$$
-D^{2}-i\left(\sigma_{\mu} \otimes 1 \otimes 1+n_{p} \sigma_{\mu} \otimes \Gamma_{p} \otimes 1+n_{\alpha} \sigma_{\mu} \otimes \Gamma_{\alpha} \otimes 1\right) B_{\mu}
$$

This acts on a combined space with $P= \pm 1$. The two linear operators $n_{p} \Gamma_{p}$ and $n_{\alpha} \Gamma_{\alpha}$ anticommute. Again, it is convenient to perform a discrete Fourier transformation to diagonalize the operator (4.15) as much as possible. The operator (4.15) becomes block diagonal, each block acting on $\left(\lambda^{r}, \psi^{r}, \lambda^{-r}, \psi^{-r}\right)$. The explicit form of each block is

$$
-D^{2}-i \sigma_{\mu} B_{\mu} \otimes\left(\begin{array}{cccc}
1+\left|n_{p}\right| & \frac{1+\cos \left(\frac{2 \pi r}{k}\right)}{2}\left|n_{\alpha}\right| & 0 & \frac{\sin \left(\frac{2 \pi r}{k}\right)}{2}\left|n_{\alpha}\right| \\
\frac{1+\cos \left(\frac{2 \pi r}{k}\right)}{2}\left|n_{\alpha}\right| & 1-\left|n_{p}\right| & -\frac{\sin \left(\frac{2 \pi r}{k}\right)}{2}\left|n_{\alpha}\right| & 0 \\
0 & -\frac{\sin \left(\frac{2 \pi r}{k}\right)}{2}\left|n_{\alpha}\right| & 1+\left|n_{p}\right| & \frac{1+\cos \left(\frac{2 \pi r}{k}\right)}{2}\left|n_{\alpha}\right| \\
\frac{\sin \left(\frac{2 \pi r}{k}\right)}{2}\left|n_{\alpha}\right| & 0 & \frac{1+\cos \left(\frac{2 \pi r}{k}\right)}{2}\left|n_{\alpha}\right| & 1-\left|n_{p}\right|
\end{array}\right) \otimes 1
$$

The four by four matrix appearing here is equal to the identity matrix plus an orthogonal matrix, and is straightforward to diagonalize. This shows that the square of the Dirac operator contains four copies of $\Delta_{r}^{+}$and four copies of $\Delta_{r}^{-}$for each $r=0, \ldots, k-1$, where

$$
\Delta_{r}^{ \pm}=-D^{2}-i\left(1 \pm \lambda_{r}\right) B_{\mu} \sigma_{\mu} .
$$

The total nonzero mode determinants are then

$$
\prod_{r=0}^{k-1} \frac{\operatorname{det}\left(\Delta_{r}^{+}\right) \operatorname{det}\left(\Delta_{r}^{-}\right)}{\operatorname{det}\left(\Delta_{r}\right)^{\frac{1}{2}} \operatorname{det}\left(-D^{2}\right)^{2}}
$$

where det is the determinant with the omission of the zero modes.

It would be interesting to complete the one-loop calculation along the lines of [13] and to compare to the supergravity result. We do expect quantitative agreement in the orbifold limit, but this clearly requires a rather nontrivial calculation in the quiver gauge theory.

Finally, we will demonstrate that the instanton calculation yields a nonzero result by showing that there are precisely eight fermionic zero modes. It is known that $\Delta_{0}^{+}$has two zeromodes and $\Delta_{0}^{-}$has no zeromodes [28], thus we need to show that the operators $\Delta_{r}^{+}$and $\Delta_{r}^{-}$have no zeromodes for $r \neq 0$. For this purpose consider the operator $D_{\lambda}=$ 
$\sigma_{\mu}\left(\partial_{\mu}+A_{\mu}\right)+i \lambda \varphi$, where $A_{\mu}$ is the monopole gauge field, $F=* D \varphi$ and $0 \leq \lambda \leq 1$. We have

$$
\begin{aligned}
\left(\lambda^{2}-1\right) \varphi^{2}+D_{\lambda}^{\dagger} D_{\lambda} & =-\left(D_{\mu} D_{\mu}+\varphi^{2}\right)-i(1+\lambda) \sigma_{\mu} B_{\mu} \\
\left(\lambda^{2}-1\right) \varphi^{2}+D_{\lambda} D_{\lambda}^{\dagger} & =-\left(D_{\mu} D_{\mu}+\varphi^{2}\right)-i(1-\lambda) \sigma_{\mu} B_{\mu} .
\end{aligned}
$$

The operator $-\left(D_{\mu} D_{\mu}+\varphi^{2}\right)$ is the operator $-D^{2}$ above. We see therefore that for $\lambda<1$, $-D^{2}-i(1 \pm \lambda) \sigma_{\mu} B_{\mu}$ is the sum of two positive semidefinite operators. The only possible zeromodes are common zeromodes of $D_{\lambda}$ or $D_{\lambda}^{\dagger}$ and $\varphi$. But such zeromodes would also be zeromodes of $-D^{2}$, which has no zeromodes. Hence, $-D^{2}+i \lambda \sigma_{\mu} B_{\mu}$ has no zeromodes for $0 \leq \lambda<2$, and neither have $\Delta_{r}^{ \pm}$for $r \neq 0$.

\section{Supergravity Computation}

For membrane scattering in flat space, the supergravity computation for the process with exchange of one unit of longitudinal momentum $\Delta p_{-}=1 / R\left(R=R_{s} \gamma\right)$ gives the following coefficient multiplying the fourth power of the transverse velocity of the membrane

$$
A_{\text {flat }}(b, R) \sim\left(\frac{1}{R^{3} b^{3}}+\frac{3}{R^{2} b^{4}}+\frac{3}{R b^{5}}\right) \exp \left(-\frac{b}{R}\right) .
$$

It was shown by Polchinski and Pouliot [13] that the leading term for $R \ll b$ agrees with the leading term in the instanton calculation in the $N=8$ gauge theory in three dimensions. (The second and the third terms between the parenthesis in the right hand side have the form of two and three-loop corrections. It would be interesting to compare these with the gauge theory computations. In particular, the above form suggests that the agreement with the supergravity computation requires a non-renormalization theorem beyond three-loop in the gauge theory computation.)

In the curved space case, we just have to replace the above expression by an appropriate Green's function ${ }^{1}$ on $S^{1} \times R^{3} \times \mathcal{M}$. When the distance between the two membranes is shorter than the typical curvature radius of $\mathcal{M}$, we can use the De Witt-Schwinger expansion to evaluate the Green's function for the exchange of $n$-units of $p_{11}$ as

$$
A_{\text {curved }}(X, Y ; R) \sim \sum_{j=0}^{\infty} a_{j}(X, Y)\left(\frac{R^{3}}{2 n^{2}} \frac{\partial}{\partial R}\right)^{j} \mathcal{A}_{\text {flat }}(\sigma(X, Y), R / n),
$$

\footnotetext{
${ }^{1}$ This can be shown by treating one membrane as a source and the other as a probe, as in [13]. Notice that because the membranes have equal mass this is only a good approximation for the leading term in the velocity expansion of the scattering amplitude.
} 
where the coefficients $a_{j}$ for the Ricci-flat Kähler manifold is given by

$$
\begin{aligned}
& a_{0}(X, Y)=1, \\
& a_{1}(X, Y)=-\frac{1}{180} R_{\alpha \beta \gamma}^{\mu} R^{\alpha \beta \gamma \nu} \partial_{\mu} \sigma^{2}(X, Y) \partial_{\nu} \sigma^{2}(X, Y), \\
& a_{2}(X, Y)=\frac{1}{180} R_{\alpha \beta \gamma \delta} R^{\alpha \beta \gamma \delta}, \quad a_{3}(X, Y)=\cdots, \cdots
\end{aligned}
$$

For $R \ll \sigma(X, Y)$, it becomes

$$
\begin{aligned}
A_{\text {curved }}(X, Y ; R) \sim & {\left[\frac{n^{3}}{R^{3} \sigma^{3}(X, Y)}+\frac{3 n^{2}}{R^{2} \sigma^{4}(X, Y)}+\frac{3 n}{R \sigma^{5}(X, Y)}+\right.} \\
& a_{1}(X, Y)\left(\frac{n^{2}}{2 R^{2} \sigma^{2}(X, Y)}-\frac{3}{2 \sigma^{4}(X, Y)}-\frac{3 R}{2 n \sigma^{5}(X, Y)}\right)+ \\
& \left.+a_{2}(X, Y)\left(\frac{n}{4 R \sigma(X, Y)}+\cdots\right)+\cdots\right] \exp \left(-n \frac{\sigma(X, Y)}{R}\right) .
\end{aligned}
$$

In the graviton scattering with zero $p_{11}$ transfer studied in $[9,10]$, the subleading terms in the adiabatic expansion (5.2) were a major source of the discrepancy between the supergravity and gauge theory computations. For finite $N$, the quantum mechanics of D0 branes cannot generate such subleading terms. The situation seems better in our case here as the subleading terms take the form of an expansion in

$$
\frac{R}{\sigma(X, Y)} \simeq \frac{e^{2}}{|\phi|},
$$

which corresponds to loop corrections in the gauge theory. As the instanton breaks all the supersymmetry, we expect loop corrections to appear to all orders in the perturbative expansion in the instanton background.

However we have already seen that the instanton action in the gauge theory computation is not equal to the geodesic distance $\sigma(X, Y)$ but is smaller than that. This means that the gauge theory amplitude is exponentially larger than the corresponding supergravity amplitude. The first non-trivial curvature dependence appears in the first order correction and is proportional to $a_{1}(X, Y)$. It would be also interesting to compare this with the gauge theory computation.

\section{Gauge Theory and Supergravity}

In this paper, we performed an instanton computation for the quiver gauge theory, which describes the dynamics of membranes in the ALE space in the low-energy shortdistance region. We found that the gauge theory amplitude is different from the supergravity amplitude. 
As far as we know, the quiver gauge theory is the unique theory in three dimensions which is renormalizable and reproduces the correct moduli space structure. In one relaxes the renormalizability condition, it would be possible to construct a model whose instanton action is exactly equal to $\frac{n}{e^{2}} \sigma(X, Y)$. The Lagrangian constructed in [16] may well have this property since its low energy action is given by (3.3) with the Euclidean distance in the last term replaced by the geodesic distance, thereby removing the error term in (3.18). However, as we noted earlier, a non-renormalizable theory cannot be used for the instanton computation if it involves quantities of order $\sigma(X, Y) / R_{c}$ or smaller, where $R_{c}$ is the typical curvature radius of $\mathcal{M}$. Since the issue is whether the instanton action $S_{\text {instanton }}$ agrees with the geodesic distance $\frac{n}{e^{2}} \sigma(X, Y)$, including the subleading terms in the expansion in $\sigma(X, Y) / R_{c}$, the computation done using the non-renormalizable model cannot be trusted and we have to rely on a renormalizable gauge theory.

What does this result imply for the large- $N$ Matrix Theory conjecture [8]? To understand the origin of the discrepancy, it is important to examine the region of validity of the gauge theory computation within the framework of the large- $N$ conjecture. For large but finite $N$, the worldvolume of the membrane has the short-distance cutoff given by

$$
\delta x=\frac{L}{\sqrt{N}},
$$

where $L$ is the size of the membrane $[8,29,30]$. As pointed out in [13], the length scale for the gauge theory on the membrane differs from that on the target spacetime by the factor of $\gamma=N l_{p}^{3} / R_{s} L^{2}$. In particular the cutoff of the gauge theory is given by

$$
\delta \tilde{x}=\gamma \delta x=\frac{\sqrt{N} l_{p}^{3}}{L R_{s}} .
$$

Since the size of the cutoff is crucial in the following discussion, we will rederive this formula in the appendix of this paper, starting from the large- $N$ Matrix Theory. The gauge theory description is applicable only if the cutoff $\delta \tilde{x}$ is smaller than the scales set by the gauge coupling constant

$$
e^{-2}=\frac{l_{s}}{g \gamma}=\frac{L^{2}}{N R_{s}}
$$

and the Compton wave-length of the W-boson

$$
|\phi|^{-1}=\frac{l_{s}^{2}}{\sigma(X, Y)}=\frac{l_{p}^{3}}{\sigma(X, Y) R_{s}} .
$$

The condition $\delta \tilde{x} \ll e^{-2}$ requires

$$
l_{p} \ll \delta x,
$$


and $\delta \tilde{x} \ll|\phi|^{-1}$ gives

$$
\sigma(X, Y) \ll \delta x
$$

The gauge theory description is valid only if both the Planck length $l_{p}$ and the distance $\sigma(X, Y)$ between the membranes are shorter than the cutoff distance $\delta x$ on the membrane. Thus the gauge theory and the supergravity cover complementary regimes and there is no overlap between the two.

The condition (6.5) also implies that the radius $R$ of the boosted M-circle is smaller than the Planck length because

$$
R=R_{s} \gamma=\frac{l_{p}^{3}}{\delta x^{2}}
$$

It is interesting to note that a membrane wrapped on the boosted M-circle becomes a string whose tension $\hat{l}_{s}^{-2}$ is equal to the cutoff scale of the membrane as

$$
\hat{l}_{s}=\sqrt{\frac{l_{p}^{3}}{R}}=\delta x .
$$

Combined with (6.5), for the gauge theory description to be valid, the length scales need to line up as

$$
R \ll l_{p} \ll \hat{l}_{s}, \quad \sigma(X, Y) \ll \hat{l}_{s}
$$

If we identify the wrapped membrane as the fundamental IIA string, this is exactly the condition for the D2-brane to be described by the gauge theory [4]. Thus we have made a full circle and came back to our starting point (1.1). Beyond this regime, we cannot ignore non-renormalizable interactions generated by massive excitations of the wrapped membrane and there is no reason to believe that the gauge theory correctly describes the membrane dynamics. The instanton computation in this paper is an explicit example of this fact. In the gauge theory regime, the effective coupling constant is given by

$$
\frac{e^{2}}{|\phi|}=\frac{N l_{p}^{3}}{L^{2} \sigma(X, Y)}=\frac{R}{\sigma(X, Y)} .
$$

Therefore the gauge theory is weakly coupled if $R \ll \sigma(X, Y)$. This condition is compatible with (6.9). We conclude that, even in the large- $N$ limit of Matrix Theory, the gauge theory description of the membrane is valid only in the short-distance regime.

Another context in which the gauge theory analysis presented in this paper may be of relevance is the scattering of gravitons in type IIB string theory compactified on an ALE space with Y-momentum transfer [31]. Here, the starting point is M-theory compactified on a two-torus times an ALE space. In the Seiberg-Sen limit $[5,6]$ we obtain after Tdualizing the two-torus an $U(N)$ three-dimensional quiver gauge theory. M-theory on $T^{2}$ 
is dual to type IIB string theory on a circle, and the magnetic flux of the three-dimensional gauge theory corresponds to the momentum along the circle (called Y-momentum in [31]). Thus exchange of Y-momentum corresponds to an instanton process in the quiver gauge theory. Repeating the analysis in this paper will once more result in a discrepancy between the gauge theory and supergravity calculation. In this case the resolution is probably again that there exists a short-distance cutoff for the three-dimensional gauge theory, so that the gauge theory only describes the short-distance regime. Massive states from wrapped branes give rise to an UV cutoff in three dimensions, in a similar way as the short-distance cutoff discussed above could be attributed to a membrane wrapped on the boosted Mcircle (see (6.8)). It would be interesting to understand this in more detail, and to see how general this phenomenon is.

\section{Acknowledgements}

HO would like to thank Joe Polchinski for discussions that initiated this work. We would also like to thank Tom Banks, Korkut Bardakci, Mike Douglas, Jeff Harvey, Shamit Kachru, Hitoshi Murayama, Steve Shenker and Stefan Vandoren for discussions.

JdB and $\mathrm{HO}$ would also like to thank the organizers of the CERN workshop, "Nonperturbative Aspects of Strings, Branes and Fields," December 8 - 12 for their hospitality. $\mathrm{KH}$ and HO thank Institute for Theoretical Physics at Santa Barbara.

This work is supported in part by NSF grant PHY-951497 and by DOE grant DEAC03-76SF00098. KH and HO are also supported in part by NSF grant PHY94-07194 through the Institute for Theoretical Physics. JdB is a fellow of the Miller Institute for Basic Research in Science. 


\section{Appendix}

\section{A Short-Distance Cutoff on Membrane}

It has been shown in $[22,23]$ that small fluctuations of membranes in the large- $N$ Matrix Theory are described by a three-dimensional gauge theory. In order to understand the region of validity of the gauge theory description, we estimate here the cutoff length of the gauge theory. For flat eleven-dimensional spacetime, $N$ D0-branes are described by the gauge theory in $(0+1)$ dimensions of [32]. The bosonic part of the action is

$$
S=\frac{1}{g_{s} l_{s}} \int \mathrm{d} t \operatorname{Tr}\left(\left(D_{0} M^{I}\right)^{2}+l_{s}^{-4}\left[M^{I}, M^{J}\right]^{2}\right) .
$$

In the case of $S^{1} \times R^{6} \times \mathcal{M}$, we use the quiver gauge theory of [17]. We treat only the case with a single membrane, but the generalization to several membranes is straightforward.

Following [8], we introduce canonical variables $p$ and $q$ with the commutation relation

$$
[q, p]=\frac{i}{N}
$$

We consider the matrices $M^{I}$ as periodic functions of $p$ and $q$ with periods 1 . The commutator and the trace are replaced as $\left[M, M^{\prime}\right] \rightarrow \frac{i}{N}\left\{M, M^{\prime}\right\}$ and $\operatorname{Tr} \rightarrow N \int_{[0,1]^{2}} \mathrm{~d} p \mathrm{~d} q$, where $\{$,$\} is the Poisson bracket. For finite N$, this is an approximation which is valid only for slowly varying functions. More precisely, the short distance cutoff is set by the uncertainty relation

$$
\delta p \delta q \sim \frac{1}{N}
$$

A flat membrane of size $L \times L$ can be realized $[8,21,22]$ as the background with

$$
M^{1}=L p, \quad M^{2}=L q, \quad M^{3}=\cdots=M^{9}=0 .
$$

Here we introduce coordinates on the membrane $x^{1}, x^{2}$ of period $L$ by $L p=x^{2}, L q=-x^{1}$. With respect to these coordinates, the bracket and the trace are expressed as

$$
\begin{aligned}
{\left[M, M^{\prime}\right] } & \longrightarrow i \frac{L^{2}}{N}\left(\frac{\partial M}{\partial x^{1}} \frac{\partial M^{\prime}}{\partial x^{2}}-\frac{\partial M}{\partial x^{2}} \frac{\partial M^{\prime}}{\partial x^{1}}\right), \\
\operatorname{Tr} & \longrightarrow \frac{N}{L^{2}} \int_{[0, L]^{2}} \mathrm{~d} x^{1} \mathrm{~d} x^{2} .
\end{aligned}
$$

Now let us consider fluctuations around the background (A.4) parametrized by spatial components of a gauge field $\left(A_{1}, A_{2}\right)$ and the transverse position $X^{i}(i=3, \ldots, 9)$ of the 
membrane as

$$
\begin{aligned}
M^{1} & =x^{2}+\frac{L^{2}}{N} A_{1}, \quad M^{2}=-x^{1}+\frac{L^{2}}{N} A_{2} \\
M^{i} & =X^{i} .
\end{aligned}
$$

The covariant derivatives and commutators are given as

$$
\begin{aligned}
D_{0} M^{i} & =\partial_{0} X^{i}-\frac{L^{2}}{N}\left(\partial_{1} A_{0} \partial_{2} X^{i}-\partial_{2} A_{0} \partial_{1} X^{i}\right) \\
{\left[M^{r}, M^{i}\right] } & =-i \frac{L^{2}}{N} \partial_{r} X^{i}+i\left(\frac{L^{2}}{N}\right)^{2}\left(\partial_{1} A_{r} \partial_{2} X^{i}-\partial_{2} A_{r} \partial_{1} X^{i}\right) \quad(r=1,2) \\
D_{0} M^{r} & =\frac{L^{2}}{N} \partial_{0} A_{r}-\left(\frac{L^{2}}{N}\right)^{2}\left(\partial_{1} A_{0} \partial_{2} A_{r}-\partial_{2} A_{0} \partial_{1} A_{r}\right) \\
{\left[M^{1}, M^{2}\right] } & =i \frac{L^{2}}{N}-i\left(\frac{L^{2}}{N}\right)^{2}\left(\partial_{1} A_{2}-\partial_{2} A_{1}\right)+i\left(\frac{L^{2}}{N}\right)^{3}\left(\partial_{1} A_{1} \partial_{2} A_{2}-\partial_{2} A_{1} \partial_{1} A_{2}\right)(\mathrm{A} .8)
\end{aligned}
$$

When we consider fluctuations of wavelengths longer than the cutoff set by the uncertainty (A.3), $\delta x^{1} \delta x^{2} \sim L^{2} / N$, the two derivative terms in the above are all negligible and we only have to keep the one derivative terms. (When we compute $\left[M^{1}, M^{2}\right]^{2}$, the product of the constant term and the two derivative term in $\left[M^{1}, M^{2}\right]$ gives a finite contribution. However it is a total derivative and does not contribute to the action.) In the long wavelength regime, (A.8) can be rewritten in terms of the curvature as

$$
D_{0} M^{r}=\left(L^{2} / N\right) F_{0 r}, \quad\left[M^{1}, M^{2}\right]=i L^{2} / N-i\left(L^{2} / N\right)^{2} F_{12} .
$$

Substituting them into (A.1) and throwing away the constant term, we obtain

$$
\begin{aligned}
S= & \frac{1}{g_{s} l_{s}} \frac{N}{L^{2}} \int \mathrm{d} t \mathrm{~d} x^{1} \mathrm{~d} x^{2}\left[\left(\partial_{0} X^{i}\right)^{2}-l_{s}^{-4}\left(\frac{L^{2}}{N}\right)^{2}\left(\partial_{r} X^{i}\right)^{2}+\left(\frac{L^{2}}{N}\right)^{2}\left(F_{0 r}\right)^{2}-l_{s}^{-4}\left(\frac{L^{2}}{N}\right)^{4}\left(F_{12}\right)^{2}\right] \\
& =\frac{l_{s}^{3}}{g_{s}} \frac{N}{L^{2}} \int \mathrm{d} t \mathrm{~d} x^{1} \mathrm{~d} x^{2}\left[\left(\partial_{0} \phi^{i}\right)^{2}-\left(\frac{L^{2}}{N l_{s}^{2}}\right)^{2}\left(\partial_{r} \phi^{i}\right)^{2}+\left(\frac{L^{2}}{N l_{s}^{2}}\right)^{2}\left(F_{0 r}\right)^{2}-\left(\frac{L^{2}}{N l_{s}^{2}}\right)^{4}\left(F_{12}\right)^{2}\right],
\end{aligned}
$$

where we have normalized the scalar field by the string tension as $\phi^{i}=l_{s}^{-2} X^{i}$. The action does not look Lorentz invariant in $(2+1)$ dimensions because of the various powers of

$$
\gamma=\frac{N l_{s}^{2}}{L^{2}}=\frac{N l_{p}^{3}}{L^{2} R_{s}}
$$

that appear in it. In fact, these different powers can be removed by rescaling the spatial coordinates on the membrane [13] as

$$
\widetilde{x}^{r}=\gamma x^{r}
$$


After the rescaling, we have

$$
S=\frac{l_{s}}{g_{s} \gamma} \int \mathrm{d} t \mathrm{~d} \widetilde{x}^{1} \mathrm{~d} \widetilde{x}^{2}\left(\left(\partial_{0} \phi^{i}\right)^{2}-\left(\partial_{\tilde{r}} \phi^{i}\right)^{2}+\left(F_{0 \tilde{r}}\right)^{2}-\left(F_{\tilde{1} \tilde{2}}\right)^{2}\right),
$$

where the spatial integration is over $[0, \gamma L] \times[0, \gamma L]$. From this we see that the gauge coupling constant is given by

$$
e^{2}=\frac{g_{s} \gamma}{l_{s}}
$$

The uncertainty relation (A.3) is translated to the coordinates $\widetilde{x}^{r}$ as $\delta \widetilde{x}^{1} \delta \widetilde{x}^{2} \sim \gamma^{2} L^{2} / N$ which implies

$$
\delta \widetilde{x}^{r} \sim \gamma \frac{L}{\sqrt{N}}=\frac{\sqrt{N} l_{p}^{3}}{L R_{s}} .
$$

Since the mass of the membrane is $l_{p}^{-3} L^{2}$ the M-momentum is given by

$$
p_{11}=\frac{N}{R_{s}}=\left(l_{p}^{-3} L^{2}\right) \frac{v_{11}}{\sqrt{1-v_{11}^{2}}},
$$

where $v_{11}$ is the velocity in the eleven-th direction. Therefore, $\gamma=N l_{p}^{3} / R_{s} L^{2}$ can be identified as the Lorentz boost factor $\left(1-v_{11}^{2}\right)^{-\frac{1}{2}}$ in the limit $v_{11} \sim 1$.

\section{References}

[1] S. H. Shenker, "Another Length Scale in String Theory?" hep-th/9509132.

[2] J. Polchinski, "Dirichelet Branes and Ramond-Ramond Charges," Phys. Rev. Lett. 75 (1995) 4724; hep-th/9510017.

[3] U.H. Danielsson, G. Ferretti and B. Sundborg, "D Particle Dynamics and Bound States," Int. J. Mod. Phys. A11 (1996) 5463; hep-th/9603081; D. Kabat and P. Pouliot, "A Comment on Zero-brane Quantum Mechanics," Phys. Rev. Lett. 77 (1996) 1004; hep-th/96030127.

[4] M. R. Douglas, D. Kabat, P. Pouliot and S. H. Shenker, "D-branes and Short Distances in String Theory," Nucl. Phys. B485 (1997) 85; hep-th/9608024.

[5] A. Sen, "D0 Brans on $T^{n}$ and Matrix Theory," hep-th/9709220.

[6] N. Seiberg, "Why is the Matrix Model Correct?" Phys. Rev. Lett. 79 (1997) 3577; hepth/9710009.

[7] C. Bachas and M. Porrati, "Pair Creation of Open Strings in an Electric Field," Phys. Lett. 298B (1992) 77; hep-th/9209032; C. Bachas, "D-brane Dynamics," Phys. Lett. B374 (1996) 37; hep-th/9511043; M. R. Douglas and M. Li, "D-brane Realization of $N=2$ Super Yang-Mills Theory in Four Dimensions," hep-th/9604041; C. Bachas and E. Kiritsis, "F $F^{4}$ 
Terms in $N=4$ String Vacua," Nucl. Phys. Proc. Suppl. 55B (1997) 194; hep-th/9611205; K. Becker and M. Becker, "A Two-Loop Test of M(atrix) Theory", Nucl. Phys. B506 (1997) 48; hep-th/9705091.

[8] T. Banks, W. Fischler, S. H. Shenker and L. Susskind, "M Theory as a Matrix Model: A Conjecture," Phys. Rev. D55 (1997) 5112; hep-th/9610043.

[9] M. R. Douglas, H. Ooguri and S. H. Shenker, "Issues in M(atrix) Theory Compactification," Phys. Lett. B402 (1997) 36; hep-th/9702203.

[10] M. R. Douglas and H. Ooguri, "Why Matrix Theory is Hard," to appear in Phys. Lett. B; hep-th/9710178.

[11] M. Dine and A. Rajaraman, "Multigraviton Scattering in the Matrix Model," hep$\operatorname{th} / 9710174$.

[12] S. Hellerman and J. Polchinski, "Compactification in the Lightlike Limit," hep-th/9711037.

[13] J. Polchinski and P. Pouliot, "Membrane Scattering with M-Momentum Transfer," Phys. Rev. D56 (1997) 6601; hep-th/9704029.

[14] M. R. Douglas, "D-brane in Curved Space," to appear in Adv. Theor. Math. Phys.; hepth/9703056.

[15] M. R. Douglas, "D-branes and Matrix Theory in Curved Space," talk given at Strings '97; hep-th/9707228.

[16] M. Douglas, A.. Kato and H. Ooguri, "D-brane Actions on Kähler Manifolds," to appear in Adv. Theor. Math. Phys.; hep-th/9708012.

[17] M. Douglas and G. Moore, "D-branes, Quivers, and ALE Instantons," hep-th/9603167.

[18] J. Polchinski, "Tensors from K3 Orientifolds," Phys. Rev. D55 (1997) 6423; hepth/9606165.

[19] K. Intriligator and N. Seiberg, "Mirror Symmetry in Three-Dimensional Gauge Theories," Phys. Lett. B387 (1996) 513; hep-th/9607207.

[20] J. de Boer, K. Hori, H. Ooguri and Y. Oz, "Mirror Symmetry in Three-Dimensional Gauge Theories, Quivers and D-branes", Nucl. Phys. B493 (1997) 101; hep-th/9611063.

[21] B. de Wit, J. Hoppe and H. Nicolai, "On the Quantum Mechanics of Supermembranes," Nucl. Phys. B305 (1988) 545.

[22] T. Banks, N. Seiberg and S. Shenker, "Branes from Matrices," Nucl. Phys. B490 (1997) 91; hep-th/9612157.

[23] E. Keski-Vakkuri and P. Kraus, "Notes on Branes in Matrix Theory," hep-th/9706196.

[24] A. Hanany and E. Witten, "Type IIB Superstrings, BPS Monopoles, And ThreeDimensional Gauge Dynamics", Nucl. Phys. B492 (1997) 152; hep-th/9611230. 
[25] J. de Boer, K. Hori, H. Ooguri, Y. Oz and Z. Yin, "Mirror Symmetry in Three-Dimensional Gauge Theories, SL(2,Z) and D-Brane Moduli Spaces", Nucl. Phys. B493 (1997) 148; hepth/9612131.

[26] E. Corrigan and P. Goddard, "An $N$ Monopole Solution with $4 N-1$ Degrees of Freedom," Commun. Math. Phys. 80 (1981) 575.

[27] N. Dorey, V.V. Khoze, M.P. Mattis, D. Tong and S. Vandoren, "Instantons, ThreeDimensional Gauge Theory, and the Atiyah-Hitchin Manifold", Nucl.Phys. B502 (1997) 59 ; hep-th/9703228.

[28] C. Callias, "Index Theorems on Open Spaces", Commun. Math. Phys. 62 (1978) 213.

[29] O. Aharony and M. Berkooz, "Membrane Dynamics in M(atrix) Theory," Nucl. Phys. B491 (1997) 184; hep-th/9611215.

[30] D. Kabat and W. Taylor, "Spherical Membranes in Matrix Theory," hep-th/9711078.

[31] T. Banks, W.Fischler, N. Seiberg, and L. Susskind, "Instantons, Scale Invariance and Lorentz Invariance in Matrix Theory", Phys. Lett. B408 (1997) 111; hep-th/9705190.

[32] M. Claudson and M. B. Halpern, "Supersymmetric Ground State Wave Functions," Nucl. Phys. B250 (1985) 689. 